\title{
Froissart bound and gluon number fluctuations
}

\author{
Wenchang Xiang* \\ Department of Physics, The University of South Dakota, Vermillion, SD 57069, USA \\ Fakultät für Physik, Universität Bielefeld, D-33501 Bielefeld, Germany
}

(Dated: October 31, 2018)

\begin{abstract}
We study the effect of gluon number fluctuations (Pomeron loops) on the impact parameter behavior of the scattering amplitude in the fixed coupling case. We demonstrate that the dipolehadron cross-section computed from gluon number fluctuations saturates the Froissart bound and the growth of the radius of the black disk with rapidity is enhanced by an additional term as compared to the single event case. We find that the physical amplitude has a Gaussian impact parameter dependence once the gluon number fluctuations are included. This indicates that the fluctuations may be the microscopic origin for the Gaussian impact parameter dependence of the scattering amplitude.
\end{abstract}

PACS: $11.55 . \mathrm{Bq}, 13.60 . \mathrm{Hb}$

\section{INTRODUCTION}

The consistent description of the impact parameter behavior of the scattering amplitude is a long standing problem. In this work we discuss the influence of gluon number fluctuation on this behavior.

In fact, some activities toward understanding how fluctuations change the impact parameter dependence of the scattering amplitude have already started (see for example Refs. 1-3]). Nevertheless, it is necessary to admit that we are still far away from a complete and consistent theory related to this subject. The most crucial difficulty related to the impact parameter dependence is the non-perturbative (soft) contribution, which should be taken into account at large values of the impact parameter (for reviews see [4 [6] and references therein). In our approach we use a different technique compared to Refs. [1-3]. Namely, we calculate the rapidity dependence of the radius of the black disk in the fluctuation-dominated (diffusive scaling) region at high energy.

The gluon number fluctuations become important at very high energy. Therefore, when considering the way how the Froissart bound may emerge based on the knowledge gathered in the small-x physics, the effects of the most recent elements in the evolution and the effects of Pomeron loop, have to be taken into account.

In this work we focus on the consequences of fluctuations on the impact parameter dependence of the scattering amplitude. In Section [I and III, we will briefly review the Froissart bound and the non-perturbative input of the scattering amplitude. The Froissart bound including gluon saturation effects will be studied in Section IV] It turns out that the total cross section saturates the Froissart bound in the case of gluon saturation. In Section V , we will compute the impact parameter dependence of the physical amplitude including gluon number fluctuations. We find that the physical amplitude has a Gaussian dependence on the impact parameter, which is in agreement with experimental measurements. We also calculate the radius of the black disk including gluon number fluctuations and find a unique rapidity dependence coming from fluctuations. Further, in Section VI we calculate the slope parameter $B$. The summary is given in Section VII.

\section{UNITARITY AND FROISSART BOUND}

In high energy scattering processes, the total cross section for the reaction $12 \longrightarrow n$ particles is

$$
\sigma_{12 \longrightarrow n}=\frac{1}{4\left|\mathbf{p}_{\mathbf{1}}\right| \sqrt{\mathbf{s}}} \sum(2 \pi)^{4} \delta^{4}\left(P^{f}-P^{i}\right)\left|\left\langle f_{n}|T| i\right\rangle\right|^{2} .
$$

Here $\left|\mathbf{p}_{\mathbf{1}}\right|$ is the magnitude of the initial center-of-mass frame three momentum. It is well known that the probability conservation in the scattering processes requires the scattering $S$ matrix to be a unitary matrix $S S^{\dagger}=1$. Unitarity of $S$ matrix provides a simple way to derive total cross sections from the forward $\left(\theta_{s}=0\right)$ elastic scattering amplitude,

*wxiangphys@gmail.com 
which is known as the optical theorem. For any orthonormal states $|i\rangle$ and $\langle j|$, one has

$$
\delta_{j i}=\left\langle j\left|S S^{\dagger}\right| i\right\rangle=\sum_{f}\langle j|S| f\rangle\left\langle f\left|S^{\dagger}\right| i\right\rangle
$$

where we have used the completeness relation

$$
\sum_{f}|f\rangle\langle f|=1
$$

We define the $T$ matrix as $S=1-i T$, then the unitarity condition requires that

$$
\langle j|T| i\rangle-\left\langle j\left|T^{\dagger}\right| i\right\rangle=(2 \pi)^{4} i \sum_{f} \delta^{4}\left(P^{f}-P^{i}\right)\left\langle j\left|T^{\dagger}\right| f\right\rangle\langle f|T| i\rangle .
$$

In the case of $j=i$, where the final state is the same as the initial state, we obtain the optical theorem

$$
\sigma_{12}^{t o t}=\frac{1}{2\left|\mathbf{p}_{\mathbf{1}}\right| \sqrt{\mathbf{s}}} \operatorname{Im}\langle i|T| i\rangle
$$

with

$$
2 \operatorname{Im}\langle i|T| i\rangle=\operatorname{Im} A(s, t=0)=\sum_{f}(2 \pi)^{4} i \delta^{4}\left(P^{f}-P^{i}\right)|\langle f|T| i\rangle|^{2},
$$

where $A(s, t)$ is the elastic scattering amplitude, $s$ and $t$ are the center-of-mass energy squared and the momentum transfer squared, respectively.

In high energy physics, the two particles scattering amplitude $A\left(s, \cos \theta_{s}\right)$ can be expanded in the partial-wave series,

$$
A\left(s, \cos \theta_{s}\right)=16 \pi \sum_{l=0}^{\infty}(2 l+1) A_{l}(s) P_{l}\left(\cos \theta_{s}\right),
$$

where $P_{l}\left(\cos \theta_{s}\right)$ is the Legendre polynomial of the first kind, and $\theta_{s}$ is the $s$ channel scattering angle in the centerof-mass frame. The partial-wave amplitude $A_{l}(s)$ can be written in terms of a real phase shift $\delta_{s}$ and an inelastic threshold $\eta_{l}$

$$
A_{l}(s)=\frac{\eta_{l}(s) e^{2 i \delta_{l}(s)}-1}{2 i \rho(s)},
$$

where $\rho(s)=2\left|\mathbf{p}_{\mathbf{1}}\right| / \sqrt{\mathbf{s}}$ with our choice of normalization, and unitarity requires that $0<\eta_{l}<1$. $A_{l}(s)$ will be exponentially small for

$$
l \geq \alpha M^{-1} \sqrt{s} \ln (s)
$$

and the scattering amplitude (7) may be truncated at this value [7]. With the unitarity constrain $0<\eta_{l}<1$ and (8), we can get

$$
\left|A_{l}(s)\right|=\left|\frac{\eta_{l}(s) e^{2 i \delta_{l}(s)}-1}{2 i \rho(s)}\right| \leq \frac{1}{\rho(s)}
$$

where $\rho(s) \longrightarrow 1$ as $s \longrightarrow \infty$. We know that the Legendre polynomial of the first kind $\left|P_{l}\left(\cos \theta_{s}=1\right)\right| \leq 1$. So, for large $s$

$$
\left|A\left(s, \cos \theta_{s}=1\right)\right| \leq \sum_{l=0}^{l_{m}}(2 l+1)
$$

with $[8]$

$$
l_{m}=\alpha M^{-1} \sqrt{s} \ln (s) .
$$


Performing the summation over $l$ in (11) gives

$$
\left|A\left(s, \cos \theta_{s}=1\right)\right| \leq \text { constat } \times s \ln ^{2}(s) .
$$

Substituting it into (5), the Froissart bound is [9]

$$
\sigma^{t o t} \leq \text { constant } \times \ln ^{2}(s)
$$

where we have used

$$
\left|\mathbf{p}_{\mathbf{1}}\right|^{2} s=\left(P_{1} \cdot P_{2}\right)^{2}-m_{1}^{2} m_{2}^{2}=\frac{1}{4}\left[s-\left(m_{1}+m_{2}\right)^{2}\right]\left[s-\left(m_{1}-m_{2}\right)^{2}\right] .
$$

Here, $P_{1}$ and $P_{2}$ are the magnitude of the initial center-of-mass frame four momentum in the two-body scattering process $1+2 \longrightarrow 3+4$, the $m_{1}$ and $m_{2}$ are the mass of particle 1 and particle 2 . The Froissart bound is one of the outstanding results of the analytic $S$ matrix theory. This bound has been derived in 1961 by Froissart 9$]$ assuming that the two particles scattering amplitude has uniformly bounded partial wave amplitudes and satisfies the Mandelstam representation with a finite number of subtractions. The Froissart bound expresses that the hadronic total cross section can not rise faster than constant $\times \ln ^{2} s$.

\section{THE NON-PERTURBATIVE INPUT}

In many practical applications, it is too complicated to perform calculations of the scattering amplitude keeping precisely the information about the impact parameter dependence, since it is related to the non-perturbative physics. In order to simplify the situation, one considers the scattering at fixed impact parameter and then introduces the knowledge about the impact parameter dependence through some profile function, which we will denote by $S(b)$. Usually, the following two ansätze are used as a non-perturbative input:

1. The scattering amplitude expressed as the product of the scattering amplitude at fixed impact parameter times the profile function $S(b)$

$$
T(Y, r, b)=T(Y, r) \cdot S(b) .
$$

Such factorization form is usually used in the region of large values of the impact parameter $b$.

2. The second one is mostly inspired by the numerical study of BK equation with a modified BK kernel in which the kernel of the BK integral equation is regulated to cut off infrared singularities [10]. The impact parameter dependence is introduced through the saturation scale, $Q_{s}(Y, b)=Q_{s}(Y) \cdot S(b)$, and consequently for the scattering amplitude we have:

$$
T(Y, Q, b)=T\left(Q, Q_{s}(Y, b)\right)=T\left(Q, Q_{s}(Y, b=0) \cdot S(b)\right) .
$$

In both cases, the impact parameter profile function typically has the exponential behavior $S(b)=e^{-2 m_{\pi} b}$ at large distances $b \gg R_{0}$, where $R_{0}$ is the typical radial size of the hadron under consideration and $R_{0}$ increases as $A^{1 / 3}$ for a nucleus with atomic number $A$. We use such an exponential fall-off at large impact parameter as a non-perturbative initial condition at low energy.

\section{SINGLE EVENT AMPLITUDE}

In the geometric scaling region and in the fixed coupling case, the dipole-hadron scattering amplitude reads

$$
T(Y, r, b) \simeq\left(r^{2} Q_{s}^{2}(Y)\right)^{\gamma_{s}} \cdot S(b),
$$

where the saturation momentum is

$$
Q_{s}^{2}(Y)=Q_{0}^{2} e^{\omega \bar{\alpha}_{s} Y}
$$


with the arbitrary reference scale $Q_{0}\left(Q_{0} \sim \mathcal{O}\left(\Lambda_{Q C D}\right)\right)$ and with the $S(b)$ giving the impact parameter dependence. Note that here factorization is assumed, which is the case as in Refs. 10, 11]. Eq. (18) shows geometric scaling with the anomalous dimension

$$
\gamma=1-\gamma_{s} \simeq 0.37
$$

Now, with the non-perturbative input

$$
S(b) \simeq e^{-2 m_{\pi} b}
$$

at large $b$, one obtains from the condition

$$
T(Y, r, R)=\kappa \simeq \mathcal{O}(1)
$$

the "black disc radius"

$$
R \simeq \frac{\gamma_{s}}{2 m_{\pi}}\left(\omega \bar{\alpha}_{s} Y-\ln \left(\frac{Q^{2}}{Q_{0}^{2}}\right)\right) .
$$

Eq. (23) gives the standard result given in the literature [11]. We have gone through such a detailed derivation of (23) since one of the main purposes of the present work is to show how Eq. (23) is modified once gluon number fluctuation effects are included.

The resulting dipole-hadron cross section saturates the Froissart bound

$$
\begin{aligned}
\sigma^{t o t}= & 2 \int d^{2} b T(Y, r, b) \\
& =2 \pi R^{2} \\
& \simeq \frac{2 \pi \gamma_{s}^{2}}{4 m_{\pi}^{2}}\left(\omega \bar{\alpha}_{s} Y-\ln \left(\frac{Q^{2}}{Q_{0}^{2}}\right)\right)^{2} \\
& \sim \frac{\pi \gamma_{s}^{2}}{2}\left(\frac{\omega \bar{\alpha}_{s}}{m_{\pi}}\right)^{2} \ln ^{2} s
\end{aligned}
$$

with $Y=\ln \left(s / Q^{2}\right)$.

We would like to note that there was some controversy between [11] and 12, 13]. The authors of Refs. 12, 13] claim that the exponential fall-off with $b$ of the initial distribution should replace by a power law fall-off due to perturbative nature of BK equation, which would be then too slow to satisfy the Froissart bound. While Ref. 11] points out that the BK equation will preserve the exponential tails at very high energy due to a quasi-locality of the BK equation. The numerical study of the BK equation with a modified BK kernel shows that the exponential tail is preserved when the kernel of the BK equation is properly regulated in the infrared[10].

It is easy to check that both ansätze in Eq. (16) and Eq. (17) for the single event amplitude lead to the similar result in Eq. (23).

\section{INCLUDING GLUON NUMBER FLUCTUATIONS}

After including fluctuations one has to distinguish between the even-by-event amplitude and the averaged (physical) amplitude. They can be explained by considering the evolution of a hadron from $y=0$ up to $y=Y$ which is probed by a dipole of size $r$, giving the amplitude $T(r, Y)$. The evolution of the hadron is stochastic and leads to random gluon number realizations inside the hadron at $Y$, corresponding to different events in an experiment. The physical amplitude, $\bar{T}(r, Y)$, is then given by averaging over all possible gluon number realizations/events, $\bar{T}(r, Y)=\langle T(r, Y)\rangle$ , where $T(r, Y)$ is the amplitude for the dipole $r$ scattering off a particular realization of the evolved hadron at $Y$.

Based on the high energy QCD/statistical physics correspondence, we can write 14]

$$
\bar{T}\left(\rho, \rho_{s}(Y, b)\right)=\left\langle T\left(\rho, \rho_{s}(Y, b)\right)\right\rangle=\int d \rho_{s}(Y, b) T\left(\rho-\rho_{s}(Y, b)\right) P\left(\rho_{s}(Y, b)-\left\langle\rho_{s}(Y, b)\right\rangle\right)
$$

where we have used $\rho=\ln \left(Q^{2} / Q_{0}^{2}\right)$ and $\rho_{s}(Y, b)=\ln \left(Q_{s}^{2}(Y, b) / Q_{0}^{2}\right)$ and we have assumed the dependence of the scattering amplitude on the impact parameter through saturation scale 15]. The probability distribution of $\rho_{s}(Y, b)$ is argued to have Gaussian form [16],

$$
P\left(\rho_{s}(Y, b)\right) \simeq \frac{1}{\sqrt{\pi D Y}} \exp \left[-\frac{\left(\rho_{s}(Y, b)-\left\langle\rho_{s}(Y, b)\right\rangle\right)^{2}}{D Y}\right]
$$


and the single scattering amplitude $T\left(\rho-\rho_{s}(Y, b)\right)$ is

$$
T\left(\rho, \rho_{s}(Y, b)\right)= \begin{cases}1 & \text { for } \rho \leq \rho_{s}(Y, b) \\ \exp \left[-\gamma_{s}\left(\rho-\rho_{s}(Y, b)\right)\right] & \text { for } \rho \geq \rho_{s}(Y, b) .\end{cases}
$$

It is easy to show that in the diffusive scaling region, $\sigma \ll \rho-\left\langle\rho_{s}(Y, b)\right\rangle \ll \gamma_{s} \sigma^{2}$,

$$
\langle T(Y, r, b)\rangle \simeq e^{-\left(\rho-\left\langle\rho_{s}(Y, b)\right\rangle\right)^{2} / D Y} \cdot \frac{\sqrt{D Y}}{\rho-\left\langle\rho_{s}(Y, b)\right\rangle}
$$

where we have used $\sigma^{2}=D Y$ with $D$ being the diffusion coefficient. Now, with the non-perturbative input

$$
\left\langle\rho_{s}(Y, b)\right\rangle \simeq \rho_{s}(Y, b) \simeq \omega \bar{\alpha}_{s} Y-2 m_{\pi} b
$$

one can easily see that the exponential decrease with $b$ in the single event case is turned into a Gaussian $b$ dependence

$$
\begin{aligned}
\langle T(Y, r, b)\rangle & \simeq \frac{\sqrt{D Y}}{\rho-\left\langle\rho_{s}(Y, b)\right\rangle} \cdot e^{-\left(\rho-\omega \bar{\alpha}_{s} Y+2 m_{\pi} b\right)^{2} / D Y} \\
& \propto \frac{1}{2 \sqrt{\pi}} e^{-\frac{4 m_{\pi}^{2} b^{2}}{D Y}} .
\end{aligned}
$$

This consequence of fluctuations seems to be supported by the experimental observations [17 19] since, say for pp collision,

$$
\frac{d \sigma_{e l}}{d t} \sim e^{-B|t|}
$$

which after a Fourier transform gives

$$
S(b) \sim e^{-\frac{b^{2}}{2 B}}
$$

Second consequence of fluctuations is that the factorization is broken, see Eq. (28),

$$
\langle T(Y, r, b)\rangle \neq f(Y, r) \cdot S(b)
$$

as compared to the single event amplitude in Eq. (18) where

$$
\langle T(Y, r, b)\rangle=f(Y, r) \cdot S(b) .
$$

Third consequence of fluctuations is that also the averaged amplitude in Eq. (30) satisfies the Froissart bound. Namely, from the condition $\langle T\rangle=\kappa \simeq \mathcal{O}(1)$ (but $\kappa \leq 1)$,

$$
\kappa \simeq \frac{\sqrt{D Y}}{\rho-\left\langle\rho_{s}(Y, R)\right\rangle} \cdot e^{-\left(\rho-\omega \bar{\alpha}_{s} Y+2 m_{\pi} R\right)^{2} / D Y}
$$

which after taking the logarithm on both sides reads ( $\kappa^{\prime}$ close to one)

$$
-\kappa^{\prime}=-\frac{\left(\rho-\left\langle\rho_{s}(Y, R)\right\rangle\right)^{2}}{D Y}+\ln \left(\frac{\sqrt{D Y}}{\rho-\left\langle\rho_{s}(Y, R)\right\rangle}\right)
$$

and is fullfilled if

$$
\rho-\left\langle\rho_{s}(Y, R)\right\rangle \simeq c \sqrt{D Y}
$$

with the coefficient $c$ of order $\sqrt{\kappa^{\prime}}$.

Now, with $\left\langle\rho_{s}(Y, R)\right\rangle \simeq \omega \bar{\alpha}_{s} Y-2 m_{\pi} R$, one obtains

$$
\begin{aligned}
\rho-\omega \bar{\alpha}_{s} Y+2 m_{\pi} R & =c \sqrt{D Y} \\
\Longrightarrow R & =\frac{1}{2 m_{\pi}}\left(\omega \bar{\alpha}_{s} Y+c \sqrt{D Y}-\ln \left(\frac{Q^{2}}{Q_{0}^{2}}\right)\right) .
\end{aligned}
$$

As compared to Eq. (23), this equation taking fluctuations into account contains a new term $\sqrt{D Y}$.

So, including fluctuations and the impact parameter dependence in the way presented here seem to lead to reasonable results. However, the whole discussion is valid only in the fixed coupling case. We have already calculated the dipolehadron scattering amplitude by considering both gluon number fluctuations and runing coupling effect [20]; however we do not know how to take into account the impact parameter dependence of the scattering on top of gluon number fluctuations and runing coupling effect. This will be considered in our next work. 


\section{PHENOMENOLOGICAL APPLICATIONS AND ESTIMATION OF THE SLOPE PARAMETER $B$}

It is well known from numerous hadronic scattering experiments that

$$
\frac{d \sigma_{\exp }}{d t} \propto e^{-B|t|}
$$

where $t$ is the squared four momentum transfer between the projectile and target. The $t-$ slope $B$ tends to a universal value determined by the proton shape alone [21]. From the experimental measurement of the $t$-distribution of the vector mesons, the effective slope $B$ is found to be $B=4 \mathrm{GeV}^{-2}$ [17 19 .

In order to study the $t$-slope $B$, we take the Fourier transform of Eq.(39):

$$
S_{\text {exp }}(b) \propto \frac{1}{2 \pi B} e^{-\frac{b^{2}}{2 B}} .
$$

Now from comparison of factors in the exponent of Eq.(30) with Eq.401, we can immediately see that

$$
\begin{aligned}
B & =\frac{\sigma^{2}}{8 m_{\pi}^{2}} \\
& =\frac{D Y}{8 m_{\pi}^{2}} \\
& =\frac{D}{8 m_{\pi}^{2}} \ln \left(\frac{s}{Q^{2}}\right)
\end{aligned}
$$

where $D$ is the diffusion coefficient. The value of $D=0.325$ is determined by fitting the HERA data with the color glass condensate model plus the gluon number fluctuations [22, 23]. Note that the $B$ increases logarithmically with the center of mass energy $s$, which is in agreement with the Regge theory. This is the phenomenon known as the shrinkage of the diffraction peek in Regge theory, which can be interpreted as an increase of the interaction radius $R_{\text {int }} \sim \sqrt{\ln s}$. With the reasonable values $m_{\pi}=0.14 G e V, \sqrt{s}=90 G e V$ and $Q^{2}=10 G e V^{2}$, one obtains values $[17-19]$

$$
\begin{aligned}
B & =\frac{D}{8 m_{\pi}^{2}} \ln \left(\frac{s}{Q^{2}}\right) \\
& \simeq 13.8 \mathrm{GeV}^{-2} .
\end{aligned}
$$

\section{SUMMARY}

The main results of this work can be summarized as follows: We have argued that the impact parameter behavior of the scattering amplitude in the presence of fluctuations has Gaussian-like behavior. Such behavior is in agreement with various phenomenological models. This indicates that fluctuations may be the microscopic origin for the Gaussian behavior.

Further, we have shown that the factorization of the impact parameter of the scattering amplitude is lost once the gluon number fluctuations are included.

We calculated the rapidity dependence of the radius of the black disk in the fluctuation-dominated (diffusive scaling) region at high energy. We found that, due to fluctuations, the growth of the radius of the black disk is enhanced by an additional (proportional to square root of rapidity) term.

\section{Acknowledgments}

I would like to thank Dr. Arif. Shoshi for suggesting this work and numerous stimulating discussions. Without his patient guidance, this work would not be possible. I acknowledge Edmond Iancu for discussions on these and related topics.

[1] E. Iancu, L. McLerran, Nucl. Phys. A793, (2007) 96; 
[2] Y. Hatta, A. H. Mueller, Nucl. Phys. A789, (2006) 285;

[3] E. Avsar, Y. Hatta, JHEP 09 (2008) 102;

[4] A. H. Mueller, arXiv:hep-ph/9911289,

[5] A.H. Mueller, hep-ph/0111244;

[6] E. Levin, arXiv:hep-ph/9710546,

[7] V. Barone and E. Predazzi, "High-Energy Particle Diffraction", Springer Press 2002;

[8] S. Donnachie, G. dosch, P. Landshoff and O. Nachtmann, "Pomeron Physics and QCD", Cambridge University Press 2005;

[9] M. Froissart, Phys. Rev. 123 (1961) 1053;

[10] T. Ikeda and L. McLerra, Nucl. Phys. A756 (2005) 385;

[11] E. Ferreiro, E. Iancu, K. Itakura and L. McLerran, Nucl. Phys. A710 (2002) 373;

[12] A. Konver and U. A. Wiedemann, Phys. Rev. D66 (2002)051502;

[13] A. Konver and U. A. Wiedemann, Phys. Lett. B551 (2003)311;

[14] E. Iancu, A. H. Mueller and S. Munier, Phys. Lett. B606 (2005)342;

[15] S. Munier, G. P. Salam and G. Soyez, Phys. Rev. D78 (2008)054009;

[16] C. Marquet, G. Soyez and B. W. Xiao, Phys. Lett. B639(2006) 635.

[17] S. Chekanov et al. [ZEUS Collaboration], Eur. Phys. J. C24 (2002) 345;

[18] S. Chekanov et al. [ZEUS Collaboration], Nucl. Phys. B695 (2004) 3;

[19] A. Aktas et al. [H1 Collaboration], arXiv:hep-ex/0510016;

[20] W. C. Xiang, Phys. Rev. D79 (2009)014012;

[21] H. Kowalski, L. Motyka and G. Watt, Phys. Rev. D74 (2006)074016;

[22] M. Kozlov, A. Shoshi and W. C. Xiang, JHEP 10 (2007) 020.

[23] W. C. Xiang, Nucl. Phys. A820 (2009) 303; 